REVIEW

\title{
Current state of adult intestinal transplantation in Europe
}

\author{
Mathias Clarysse ${ }^{\mathrm{a}, \mathrm{b}}$, Emilio Canovai ${ }^{\mathrm{a}, \mathrm{b}}$, \\ Tim Vanuytse ${ }^{\mathrm{b}, \mathrm{c}}$, and Jacques Pirenne $\mathrm{a}^{\mathrm{a}, \mathrm{b}}$
}

\begin{abstract}
Purpose of review
In Europe, adult intestinal transplantation (ITx) has continuously evolved since the first successful case in 1989. However, despite several recent innovations, no significant improvement in survival has been seen since 2005, illustrating the unique difficulty of transplanting the intestine. In this review, a subanalysis of adult ITx in Europe is discussed and recent publications on adult ITx in Europe are presented.
\end{abstract}

\section{Recent findings}

Increased medical and surgical arsenal in the treatment of intestinal failure reduce the need for ITx. At the same time, new indications (diffuse ischemia) have emerged. Static cold storage after vascular flush remains the gold standard but promising results are shown with additional luminal preservation.

Pretransplant embolization facilitates multivisceral transplantation. Chronic rejection remains a major difficulty to tackle and currently, liver inclusion is the only effective strategy. Treatment of graft-versus-hostdisease remains debated. Quality of life substantially improves after successful ITx. ITx becomes costeffective three years after transplantation.

\section{Summary}

ITx remains more challenging than other solid organ transplants. However, long term outcome, particularly after combined liver and ITx, is excellent and similar to other solid organ transplants. Further studies are warranted to tackle the fundamental immunobiological challenge that ITx represents.

\section{Keywords}

adult intestinal transplantation, intestinal failure, preservation, rejection, surgical innovations

\section{INTRODUCTION}

The intestine remains the most difficult organ to transplant. After briefly summarizing the historical milestones in adult intestinal transplantation (ITx) in Europe, we will report data from the International Intestinal Transplant Registry (ITR) and some recent developments from European centers. Finally, we will present our own center view on the evolution in the field.

\section{BRIEF HISTORY OF ADULT INTESTINAL TRANSPLANTATION IN EUROPE}

Of the six ITx attempted worldwide, before the cyclosporine (CsA) era, one had been performed in Paris in 1968 [1,2]. All cases failed and with the development of parenteral nutrition, interest in clinical ITx decreased. However, ITx was brought back to the lab to try to elucidate, in animal models, the challenges that this unique lymphoid and colonized organ represents. With the introduction of
CsA by Calne, it was hoped that results would improve [3]. In 1989, Deltz, from the University of Kiel, reported a successful case of ITx using a segmental graft from a living donor [4]. However, no long-term follow-up was available. Most other attempts at ITx under CsA in Europe (mostly by the pediatric team of Necker in Paris, France [5]) were defeated by rejection and infection. As a result, ITx programs in Europe once again ceased their activity. After the introduction of tacrolimus by Starzl [6],

\footnotetext{
a Abdominal Transplant Surgery and Transplant Coordination, University Hospitals Leuven; Department of Microbiology, Immunology and Transplantation, KU Leuven, 'beuven Intestinal Failure and Transplantation Center (LIFT), University Hospitals Leuven and ${ }^{\mathrm{C}}$ Gastroenterology and Hepatology, University Hospitals Leuven, Leuven, Belgium

Correspondence to Jacques Pirenne, Abdominal Transplant Surgery and Coordination, University Hospitals Leuven, Herestraat 49, 3000 Leuven, Belgium. Tel.: +320163406 03;. e-mail: Jacques.pirenne@uzleuven.be

Curr Opin Organ Transplant 2020, 25:000-000

DOI:10.1097/MOT.0000000000000731
} 


\section{KEY POINTS}

- Static cold storage of the intestine is considered the gold standard, however, further studies on luminal flush and machine perfusion should be performed.

- Pretransplant embolization in MVTx, and inclusion of the pancreas in ITx alone facilitate surgery.

- In the European cohort, the highest patient and graft survival are observed after cLi-ITx, clearly showing the protective effect of the liver on the bowel. Inclusion of the liver is currently the only effective method to prevent chronic rejection after ITx.

leading to considerable improvements in outcome, ITx programs were reestablished, first in the United States and then in Europe. In Europe, fundamental research continued to be very active under the leadership of Richard Wood - among others - exploring the complex lymphocyte trafficking between graft and host that is unique to ITx $[7,8]$. Of note, two cases of ITx between identical twins and without immunosuppression were performed at the University of Geneva and Cambridge $[9,10]$. The excellent outcome in these cases illustrates that the obstacle to ITx was not physiological (denervation, lymphatics interruption, ischemia) but immunobiological [rejection, graft-versus-host-disease (GVHD), infection].

\section{ADULT INTESTINAL TRANSPLANTATION ACTIVITY IN EUROPE}

Overall and until 18 September 2019, 414 adult ITx have been performed in Europe, in 26 centers (ITR data courtesy of Robert Venick and Julia Brennan). Of these, only 11 centers are currently active. This adult ITx activity is lower than in the United States. Of these ITx, 54\% have been isolated grafts. In contrast, in the United States, the proportion of combined liver-intestine (cLi-ITx), multivisceral and modified multivisceral transplantation (MVTx) is higher. In particular, modified MVTx, that is, MVTx without liver, has been rarely performed in Europe. Of the 414 ITx recipients, 188 are alive, most of them nutritionally independent, and with good functional outcome. Indications for adult ITx in Europe are similar to those in the rest of the world. The most frequent indications are short bowel syndrome (ischemia $>$ Crohn's disease $>$ volvulus) followed by tumor, retransplant, severe motility disorders and mucosal disease. Over the last 10 years, about $75 \%$ of the patients were at home at the time of ITx. With accumulating experience, graft survival rates have increased during three consecutive eras

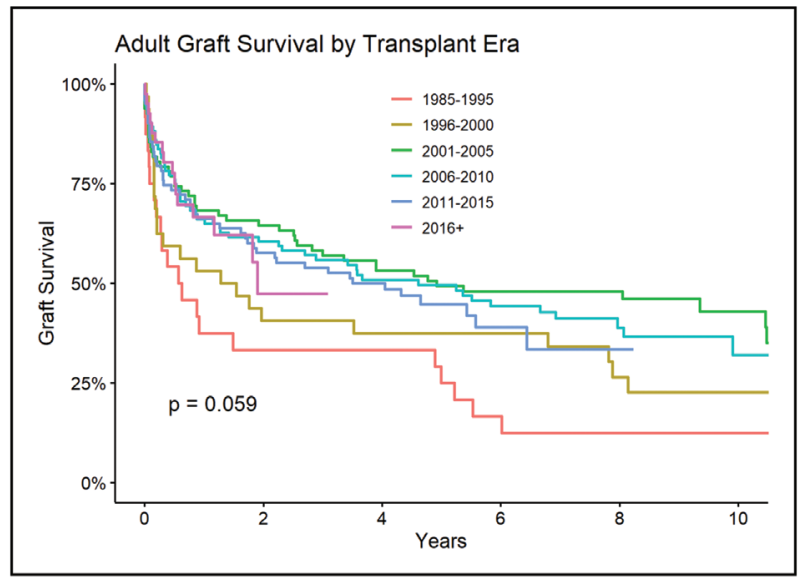

FIGURE 1. Adult graft survival in Europe, by transplant era (data collected by the ITR on 18 September 2019).

(1985-1995, 1996-2000, 2001-2005). However, no further increase in graft survival has been noted since then (Fig. 1). This clearly demonstrates that ITx is confronted with a unique biological challenge (rejection, GVHD and infection) which is not sufficiently controlled by current protocols. Nevertheless, five-year patient survival exceeds 75\% after cLiITx and is around 60\% after isolated ITx, which is similar to other solid organ transplants, whereas outcome after MVTx and modified MVTx remains inferior (Fig. 2). Of particular interest in the European cohort, not only the highest patient survival but also the highest graft survival (death-uncensored) is observed after cLi-ITx (Fig. 3). This clearly proves the protective effect of the liver on the transplanted bowel. Incidence of chronic rejection in this

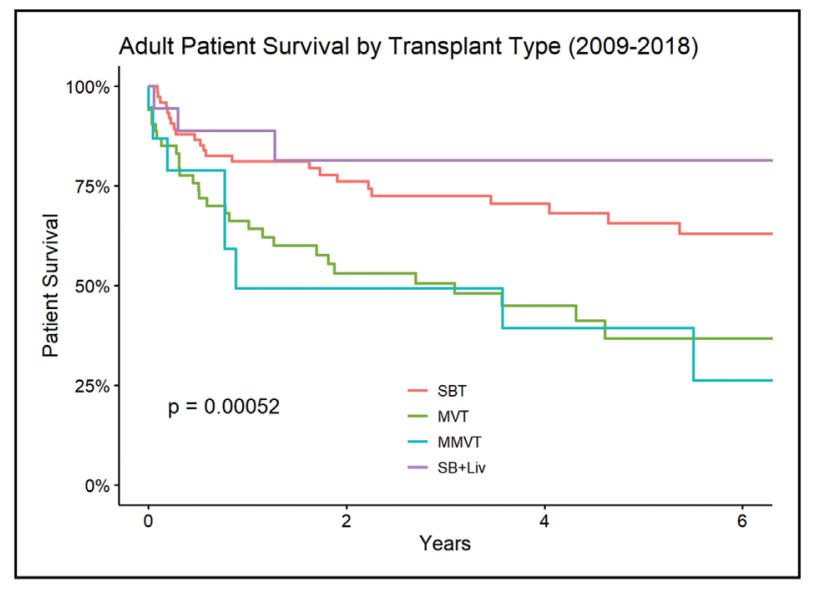

FIGURE 2. Adult patient survival in Europe, by transplant type (2009-2018) (data collected by the ITR on 18 September 2019). MMVT, modified multivisceral transplantation; MVT, multivisceral transplantation; SB+Liv, small bowel and liver transplantation; SBT, small bowel transplantation. 


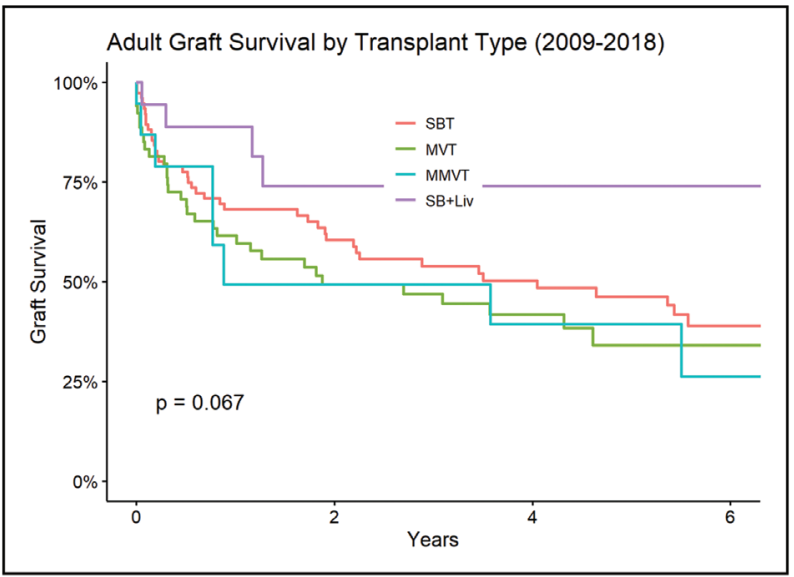

FIGURE 3. Adult graft survival in Europe, by Transplant type (2009-2018) (data collected by the ITR on 18 September 2019). MMVT, modified multivisceral transplantation; MVT, multivisceral transplantation; SB+Liv, small bowel and liver transplantation; SBT, small bowel transplantation.

group is clearly less versus isolated ITx. The results at our own center, with a five-year graft/patient survival of 91\%/91\% after cLi-ITx $(n=11)$ versus $48 \% /$ $71 \%$ after isolated ITx $(n=11)$ (unpublished data), confirm the protective effect that the liver has on the transplanted bowel.

Over the last 10 years, the annual European adult ITx activity has remained relatively stable between 10 and 25 procedures per year (Fig. 4). As in other parts of the world, the most frequent cause of morbidity and mortality are infection (often triggered by heavy chronic immunosuppression and its upscaling in case of acute rejection), malignancies (among them posttransplant lymphoproliferative disease), hypertension, diabetes, and renal failure.

\section{RECENT EUROPEAN CENTER CONTRIBUTION}

\section{Intestinal rehabilitation centers}

At the Buenos Aires ITx conference in 2015, the name of the society was changed from 'Intestinal Transplant Association' to 'Intestinal Rehabilitation and Transplant Association.' This was a response to an international appeal to merge intestinal failure and ITx care that provide all medical, nontransplant, and transplant surgical services [11]. The medical armamentarium has been enriched by new compounds [e.g. glucagon-like peptide-2 (GLP-2) analogues for short bowel syndrome and novel lipid formulations] and multidisciplinary follow-up of intestinal failure patients. Autologous intestinal reconstruction techniques [bianchi, serial transverse enteroplasty (STEP), loop reversal, and variants] have been revisited and are increasingly used $\left[12,13^{*}, 14\right]$. This has

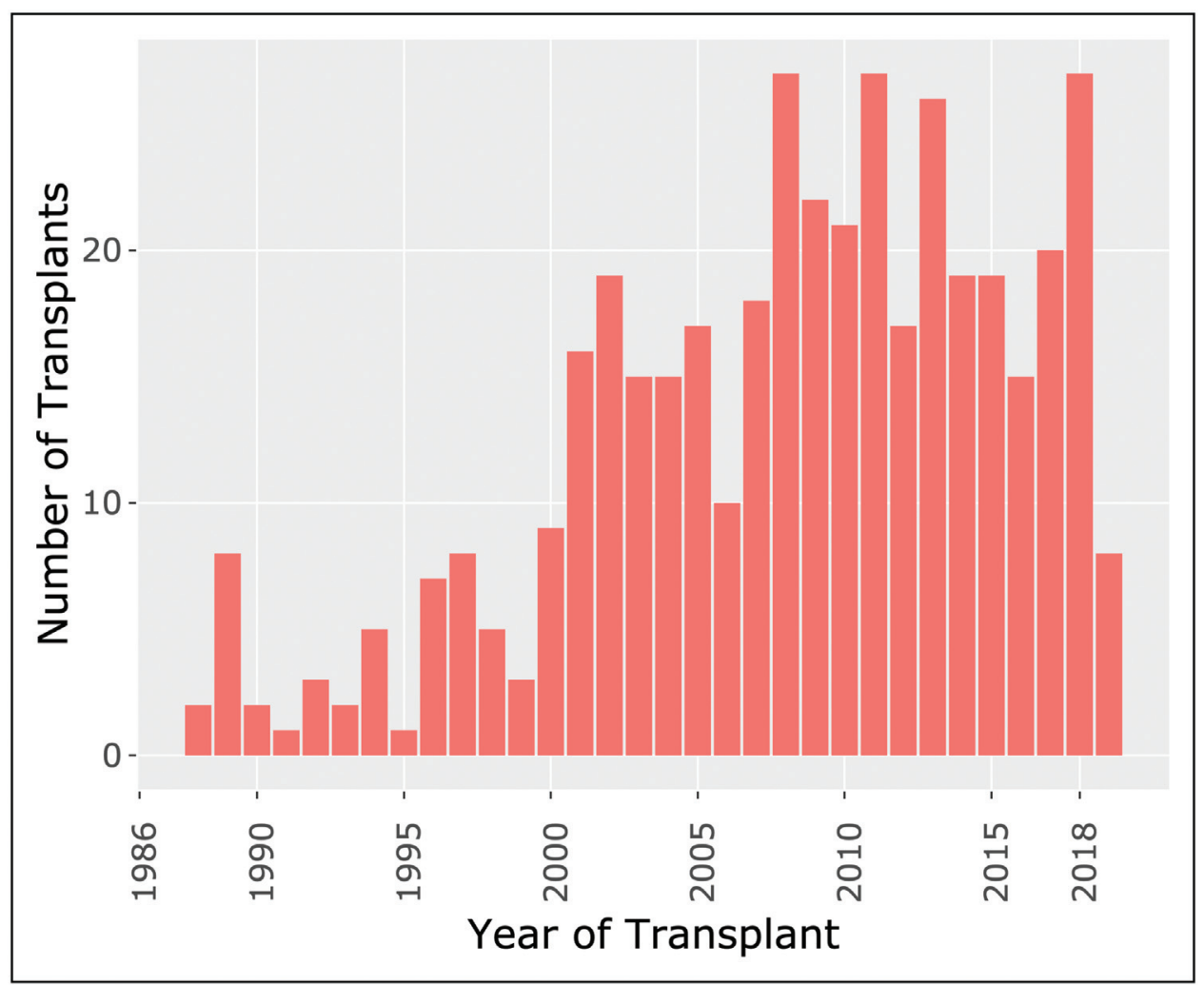

FIGURE 4: Adult intestinal transplants in Europe, per year (data collected by the ITR on 18 September 2019). 
contributed to fewer ITx in recent years worldwide. For example, Hernandez et al. [15"] reported that less than one quarter of patients who underwent intestinal lengthening required salvage ITx.

\section{Indications}

Performing ITx preemptively, that is, before the severe complications of intestinal failure have occurred, is regularly suggested. However, European gastroenterologists have remained conservative and only propose ITx as a last resort. Currently in Europe, only patients with irreversible intestinal failure and severe parenteral nutrition-related complications, including recurrent and life-threatening line infections, lack of vascular access or intestinal failure-related liver disease (IFALD) are referred for ITx $\left[12,13^{*}, 15^{\prime \prime}, 16,17^{*}, 18,19,20^{\prime \prime}\right]$.

At the same time, new indications, not necessarily associated with intestinal failure or the associated complications, have gradually emerged. For example, MVTx is being increasingly performed for diffuse venous and even arterial splanchnic thrombosis and ischemia, particularly in Cambridge $[21,22]$.

\section{Preservation}

Vascular flush with University of Wisconsin solution followed by static cold storage remains the standard preservation method. However, other preservation solutions such as histidine-tryptophanketoglutarate solution and Institut Georges Lopez (IGL-1) have been used [23]. IGL-1 has been recently introduced with success in certain European centers (Gothenburg, Groningen, Leuven, and Berlin) [24"]. This extracellular type of preservation solution (high sodium and low potassium content) contains polyethylene glycol and seems to protect the glycocalyx layer at the surface of the endothelium.

The intestinal lumen is a 'third space' and unique to the anatomy of the bowel, and some have advocated to add a luminal flush to the vascular flush. This concept has been proven efficient in several rodent experiments, probably by accelerating the cooling of the graft, washing-out feces, and possibly by a direct protective effect on the intestinal mucosa [25]. However, it is unclear which solution composition and volume is optimal. Furthermore, ITx teams have also been reluctant to perform luminal flush clinically because of the resulting bowel dilatation, complicating donor and recipient surgery.

In contrast with all other solid organs, there has been little interest in machine perfusion of the bowel. However, this may be particularly useful as the bowel is extremely sensitive to preservation and reperfusion injury. Albeit still theoretical, the possibility to 'immunomodulate' grafts during machine perfusion might be of particular interest in ITx [26]. Well-tolerated longer term preservation would also represent an additional advantage in case of complex recipient operations with anticipated long preservation periods [27"'].

\section{Surgical techniques}

\section{Abdominal wall transplantation)}

Abdominal wall transplantation (AWT) has been originally described by the Miami team in ITx patients with complete loss of abdominal domain [28]. In this procedure, the full-thickness abdominal wall is procured and transplanted with a pedicle of inferior epigastric vessels. Unsurprisingly, this adds to the complexity and duration of surgery. However, it has been performed successfully in Oxford [29] and more recently in Groningen [30]. Indications could be extended to nontransplant setting (e.g., extensive abdominal hernias, abdominal injuries or congenital malformations) [31]. AWT does not seem to be associated with an increase in intestinal rejection episodes or with the incidence of de novo donorspecific antibodies (DSA). Furthermore, the donor skin can act as a sentinel to detect rejection of the bowel, potentially allowing reduction of surveillance ITx biopsies [29,32"].

Conceptually, AWT is the best technique to restore the abdominal wall ad integrum. A simpler alternative, however, is the use of the decellularized rectus fascia that several centers now systematically retrieve during procurement as back-up [33].

\section{Embolization}

We reported that pretransplant embolization of the celiac trunk and the superior mesenteric artery greatly facilitates the extraction phase of the otherwise very high-risk MVTx operation [34]. This technique has now been applied in Cambridge, Miami, and Prague (Jiri Fronek, Prague, personal communication) $\left[35,36^{\bullet}\right]$.

Pretransplant embolization considerably decreases the perioperative risk of MVTx and opens the route to new indications previously deemed too high risk: diffuse abdominal diseases with frozen abdomen; complex third or fourth liver retransplants deemed technically too high risk for standard liver transplantation, and so on.

\section{Inclusion of pancreas}

Pancreas and intestine can be procured from a single donor and transplanted separately. However, there are no large series on the outcome of both pancreas 
and ITx from identical donors, and the safety of this technique for both recipients remains unknown. Having a sufficiently long vascular pedicle of superior mesenteric vein and artery is essential for a welltolerated implantation of the bowel graft, particularly in complex recipients. At the same time, preservation of pancreaticoduodenal branches essential for the viability of the pancreas - requires more distal dissection and transection of the mesenteric pedicle, which may necessitate complex vascular reconstruction on the bowel side. Because this adds to the complexity of an already high-risk transplant, we believe that priority should be given to the bowel. The pancreas could be used for islet retrieval instead. Per analogy with the inclusion - for technical reasons - of the pancreas in cLi-ITx grafts [37], we and other teams (Andrew Butler, Cambridge, personal communication) prefer en-bloc transplantation of pancreas and bowel instead of the bowel alone. The pancreas-bowel bloc is procured using a technique identical to the procurement of the pancreas alone, but simply leaving the small bowel and its mesentery intact and attached to it. The vascular pedicle of the bloc consists of the portal vein and an aortic patch encompassing the celiac trunk and the superior mesenteric artery. The liver can be transplanted in another recipient using the common hepatic artery. The pancreas-bowel bloc easily finds its natural position in the abdomen of the recipient. The portal vein is anastomosed end-to-side onto the recipient vena cava and the aortic patch end-to-side onto the infra-renal aorta. This makes implantation straightforward and virtually eliminates risk of torsion of the vascular pedicle. This greatly simplifies both donor and recipient surgery. The small number of adult ITx ( $\sim 20$ per year in Europe) would have a negligible impact on pancreas transplant activity (the need of which has also decreased in the last years).

\section{New insights in the immunological challenge}

Persistent inflammation and innate immune cell activation contribute to the unique challenges of ITx. Stobutzki reported the persistence of an increase in CD16+ monocytes and dendritic cells compared with healthy controls, even 10 years after transplantation [38"']. Patients with previous rejections had even higher proportions of CD16+ monocytes and dendritic cells. CD4+ and CD8+ T cells showed persistent signs of activation and the higher the donor mass, the more pronounced the increase in T-cell activation. This seems to be counterbalanced by an increased population of activated CCR4+ regulatory T cells [38"']. At our center, we found an increase in regulatory T-cell frequency in rejection-free patients under low immunosuppression [39]. In this latter report, regulatory T cells were also identified in the graft where they can probably exert their protective properties locally. Intragraft regulatory T-cells were also seen by Ruemmele in one of the longest survivors after ITx [40].

\section{Chronic rejection}

The insidious clinical presentation, the absence of noninvasive biomarkers, and the late endoscopic findings delay its diagnosis. No pharmacological therapy has proven effective and allograft removal and retransplantation remain the only options. Although acute cellular rejection can precede chronic rejection, the role of humoral immunity and DSA are gaining acceptance in the pathogenesis of chronic rejection. The impact of novel approaches, tackling antibody-mediated graft injury, remains to be seen. Moreover, chronic rejection can be seen in the absence of DSA and is not always preceded by acute rejection. Inflammatory nonimmune specific factors probably play a role.

Currently, liver inclusion appears to be the only effective strategy to prevent the development of chronic rejection. The inclusion of a liver allograft is advocated when retransplanting a sensitized recipient, because of its protective effect against humoral rejection $\left[41^{-"}, 42^{\prime \prime}\right]$.

\section{Graft-versus-host-disease}

Acute GVHD mostly develops within six months after ITx, especially after cLi-ITx and MVTx because of the higher donor lymphoid mass. One treatment is reduction or even withdrawal of immunosuppression and careful graft monitoring. Immunosuppression is then reintroduced when early signs of graft dysfunction/rejection are observed. Extracorporeal photopheresis might be an adjunct treatment for skin GVHD. Conversely, an alternative strategy is actually increasing immunosuppression. Alemtuzumab (Campath) has been reported to be efficient against GVHD in MVTx [43"']. Anti-IL2-receptor antibody and anti-tumour necrosis factor $\alpha$ antibodies have been successfully used against GVHD after liver transplantation and their effect in GVHD after ITx should be studied [44].

\section{Quality of life}

Patients being assessed for ITx have a lower quality of life (QoL) than those stable on parenteral nutrition. However, after successful ITx, QoL substantially improves. Nutritional autonomy is often achieved within three months post-ITx. However, 
many other domains continue to improve until 1224 months. Most long-term survivors are socioeconomically reintegrated, but in others, financial pressures remain, even in publicly funded healthcare systems [45"']. Compared with parenteral nutrition, ITx is cost-effective after three years, mostly because costly parenteral nutrition is no longer necessary $[46,47]$. Eight cases of pregnancies in female ITx recipients, with $100 \%$ successful live births, have been reported worldwide [48].

\section{Center view}

ITx is an extremely demanding procedure, for both physicians/allied health professionals in charge, and patients. We often say that the care of one ITx recipient takes as much time as the care of five (or more!) other solid organ transplant recipients.' Patients need to have sufficient physical and mental reserve to tolerate a complex procedure with high risk of morbidity, long hospitalizations, and frequent complications. For this reason, it is premature to routinely offer ITx preemptively based on QoLcriteria only. However, it is equally unjustified to propose ITx at a very advanced stage when the likelihood of success becomes dismal because of poor general condition and/or lack of vascular access. The latter is a situation we still unfortunately see frequently.

In our experience, pretransplant embolization makes MVTx safer, and inclusion of the pancreas in isolated ITx recipients facilitates surgery.

The major obstacle to successful ITx remains immunobiological with infection as the leading cause of death, triggered by heavy immunosuppression, particularly in case of acute rejection. Minimization of immunosuppression and therapies to facilitate graft acceptance have been shown to reduce the incidence/severity of infections, malignancies and renal failure, and to subsequently improve survival, while simultaneously limiting rejection rates [39]. In our experience, this is particularly true when the liver is simultaneously transplanted, and we believe that inclusion of the liver should be more frequently considered, and not only restricted to patients with overt IFALD.

Although acknowledging the difficulties of ITx, we believe that improved results seen at experienced centers, improved QoL, and cost-effectiveness all support the application of ITx not yet preemptively, but earlier in the course of intestinal failure.

\section{CONCLUSION}

With only 20 procedures performed annually in Europe, adult ITx is an 'orphan transplant.' Because of its rarity and high complexity, ITx should be restricted to centers with a comprehensive intestinal failure and rehabilitation program and a solid experience in other abdominal organ transplants. ITx should be offered to patients with severe intestinal failure related complications when all attempts at rehabilitation have failed, but before the window for transplantation has been lost.

\section{Acknowledgements}

We are grateful to Robert Venick, MD, and Julia Brennan, UCLA, for the subanalysis (European adult intestinal transplant) of the International Intestinal Transplant Registry.

J.P. holds a named chair at the KU Leuven of the Centrale Afdeling Voor Fractionering (CAF) and a named chair at the KU Leuven of the Institute Georges Lopez (IGL).

T.V. is a senior clinical researcher of the Flanders Research Foundation (FWO Vlaanderen, Belgium).

\section{Financial support and sponsorship}

None.

\section{Conflicts of interest}

There are no conflicts of interest.

\section{REFERENCES AND RECOMMENDED}

\section{READING}

Papers of particular interest, published within the annual period of review, have been highlighted as:

- of special interest

- of outstanding interest

1. Ruiz JO, Lillehei RC. Intestinal transplantation. Surg Clin North Am 1972 52:1075-1091.

2. Olivier C, Rettori R, Olivier C. Homotransplantation orthotopique de l'intestin grœ et des colons droit et transverse chez l'homme. J Chir 1969; 98:323.

3. Merion RM, White DJ, Thiru S, et al. Cyclosporine: five years' experience in cadaveric renal transplantation. N Engl J Med 1984; 310:148-154.

4. Deltz $E$, Schroeder $P$, Gebhardt $H$, et al. First successful clinical small intestine transplantation: tactics and surgical technic. Chirurg 1989; 60:235-239.

5. Revillon $Y$, Jan D, Goulet O, Ricour C. Small bowel transplantation in seven children: preservation technique. Transplant Proc 1991; 23:2350-2351.

6. Todo S, Tzakis AG, Abu-Elmagd $\mathrm{K}$, et al. Cadaveric small bowel and small bowel-liver transplantation in humans. Transplantation 1992; 53: 369-376.

7. Clark CL, Cunningham AJ, Crane PW, et al. Lymphocyte infiltration patterns in rat small-bowel transplants. Transplant Proc 1990; 22:2460.

8. Lear PA, Cunningham AJ, Clark CL, et al. What role for passenger leucocytes in small-bowel allografts? Transplant Proc 1990; 22:2463.

9. Morel $P$, Kadry $Z$, Charbonnet $P$, et al. Paediatric living related intestinal transplantation between two monozygotic twins: a 1-year follow-up. Lancet 2000; 355:723-724

10. Calne R, Friend $\mathrm{P}$, Middleton $\mathrm{S}$, et al. Intestinal transplant between two of identical triplets. Lancet 1997; 350:1077-1078.

11. Gondolesi GE, Fernandez A, Burghardt KM, et al. Meeting report of the XIV international small bowel transplant symposium: summary of presentations, workshops, and debates from a comprehensive meeting on intestinal failure, rehabilitation, and transplantation, Buenos Aires, Argentina. J Parenter Enter Nutr 2018; 42:477-489.

12. Lauro $A$, Lacaille F. Short bowel syndrome in children and adults: from rehabilitation to transplantation. Expert Rev Gastroenterol Hepatol 2019; 13:55-70.

13. Billiauws L, Maggiori L, Joly F, Panis $Y$. Medical and surgical management of - short bowel syndrome. J Visc Surg 2018; 155:283-291.

This article gives an overview of the medical and surgical possibilities in short bowel syndrome, before considering transplantation. 
14. Botey $\mathrm{M}$, Alastrué $\mathrm{A}$, Haetta $\mathrm{H}$, et al. Long-term results of serial transverse enteroplasty with neovalve creation for extreme short bowel syndrome: report of two cases. Case Rep Gastroenterol 2017; 11:229-240.

15. Hernandez F, Andres AM, Lopez-Santamaria M. Long-term results of surgery

- for bowel lengthening: how many transplants are avoided, for which patients? Curr Opin Organ Transplant 2018; 23:207-211.

The article analyses how successful bowel lengthening surgery can be, in preventing intestinal transplantation.

16. Marino IR, Lauro A. Surgeon's perspective on short bowel syndrome: where are we? World J Transplant 2018; 8:198-202.

17. Billiauws L, Joly F. Emerging treatments for short bowel syndrome in adult - patients. Expert Rev Gastroenterol Hepatol 2019; 13:241-246.

This study is a comprehensive review on new treatments of short bowel syndrome.

18. Lauro A, Marino IR, lyer KR. Preemptive intestinal transplant: the surgeon's point of view. Dig Dis Sci 2017; 62:2966-2976.

19. Lauro A, Pinna AD, Tossani $E$, et al. Multimodal surgical approach for adult patients with chronic intestinal pseudo-obstruction: clinical and psychosocial long-term outcomes. Transplant Proc 2018; 50:226-233.

20. Kaufman SS, Avitzur $Y$, Beath SV, et al. New insights into the indications for

-1. intestinal transplantation: consensus in the year. Transplantation 2019; 32:1.

This is a consensus/overview of the changes that have occurred in the indications

for intestinal transplantation.

21. Sharkey LM, Russell NK, Rutter CS, et al. Urgent multivisceral transplantation for widespread splanchnic ischemia. J Am Coll Surg 2016; 222:760-765.

22. Rutter CS, Amin I, Russell NK, et al. Adult intestinal and multivisceral transplantation: experience from a single center in the United Kingdom. Transplant Proc 2016; 48:468-472.

23. Mangus RS, Tector AJ, Fridell JA, et al. Comparison of histidine-tryptophanketoglutarate solution and University of Wisconsin solution in intestinal and multivisceral transplantation. Transplantation 2008; 86:298-302.

24. Canovai $\mathrm{E}$, Oltean $\mathrm{M}$, Herlenius $\mathrm{G}$, et al. First clinical multicenter experience of

- IGL-1 for intestinal graft preservation. Transplantation 2019; 103:S118.

This is the first report indicating that IGL-1 can be safely used for intestinal graft preservation.

25. Oltean M, Churchill TA. Organ-specific solutions and strategies for the intestinal preservation. Int Rev Immunol 2014; 33:234-244.

26. Muñoz-Abraham AS, Patrón-Lozano R, Narayan RR, et al. Extracorporeal hypothermic perfusion device for intestinal graft preservation to decrease ischemic injury during transportation. J Gastrointest Surg 2016; 20:313-321.

27. Weissenbacher A, Vrakas G, Nasralla D, Ceresa CD. The future of organ

I. perfusion and re-conditioning. Transpl Int 2019; 32:586-597.

This is a detailed review on intestinal graft storage solutions and machine perfusion.

28. Levi DM, Tzakis AG, Kato $T$, et al. Transplantation of the abdominal wall. Lancet 2003; 361:2173-2176.

29. Gerlach UA, Vrakas G, Sawitzki B, et al. Abdominal wall transplantation: skin as a sentinel marker for rejection. Am J Transplant 2016; 16:1892-1900.

30. Haveman JW, Tempelman TM, Hofker HS, et al. First combined intestinal and abdominal wall transplantation in the Netherlands. Ned Tijdschr Geneeskd 2016; 160:A9788.

31. Molitor $M$, Oliverius $M$, Sukop A. Abdominal wall allotransplantation. Biomed Pap 2018; 162:184-189.

32. Weissenbacher $A$, Vrakas $G$, Chen $M$, et al. De novo donor-specific HLA

- antibodies after combined intestinal and vascularized composite allotransplantation: a retrospective study. Transpl Int 2018; 31:398-407.

This is the first study looking into de-novo formation of donor-specific HLA antibodies in ITx with and without abdominal wall transplantation.
33. Gondolesi G, Selvaggi G, Tzakis A, et al. Use of the abdominal rectus fascia as a nonvascularized allograft for abdominal wall closure after liver intestinal, and multivisceral transplantation. Transplantation 2009; 87: $1884-1888$.

34. Ceulemans $L$, Jochmans I, Monbaliu D, et al. Preoperative arterial embolization facilitates multivisceral transplantation for portomesenteric thrombosis. Am Transplant 2015; 15:2963-2969.

35. Butler A, Russell N, Amin I, Cee T. Use of arterial embolisation to facilitate exenteration during multivisceral and small bowel transplantation. Transplantation 2017; 101(6S2):S25.

36. Nicolau-Raducu R, Livingstone J, Salsamendi J, et al. Visceral arterial embo-

- lization prior to multivisceral transplantation in recipient with cirrhosis, extensive portomesenteric thrombosis, and hostile abdomen: performance and outcome analysis. Clin Transplant 2019; 33:e13645.

This study gives a single-center experience on preoperative embolization in multivisceral transplantation.

37. Sudan DL, lyer KR, Deroover A, et al. A new technique for combined liver/ small intestinal transplantation. Transplantation 2001; 72:1846-1848.

38. Stobutzki N, Schlickeiser $S$, Streitz M, et al. Long-term signs of $t$ cell and myeloid

-1 cell activation after intestinal transplantation with cellular rejections contributing to further increase of CD16+ cell subsets. Front Immunol 2019; 10:1-12.

This study gives a comprehensive assessment of time-dependent and rejectiondependent alterations of systemic immune cell composition after intestinal transplantation.

39. Ceulemans L, Braza F, Monbaliu D, et al. The Leuven immunomodulatory protocol promotes T-regulatory cells and substantially prolongs survival after first intestinal transplantation. Am J Transplant 2016; 16: 2973-2985

40. Ruemmele FM, Sauvat F, Colomb V, et al. Seventeen years after successfu small bowel transplantation: long term graft acceptance without immune tolerance. Gut 2006; 55:903-904.

41. Lauro $A$, Oltean $M$, Marino IR. Chronic rejection after intestinal transplant:

-. where are we in order to avert it? Dig Dis Sci 2018; 63:551-562.

This article gives an overview on chronic rejection after intestinal transplantation and the possible strategies to tackle it.

42. Lauro A, Marino IR. Update on chronic rejection after intestinal transplant: an

- overview from experimental settings to clinical outcomes. Exp Clin Transplant 2019; 17(Suppl 1):18-30.

This is an overview on chronic rejection after intestinal transplantation.

43. Sharkey LM, Peacock S, Russell NK, et al. Graft versus host disease after

-. multivisceral transplantation: a UK center experience and update on management. Clin Transplant 2018; 32:e13239.

This study reports a single-center experience with GVHD and its management.

44. Minnee RC, Fieuws S, Jochmans I, et al. Improved survival after LTx-associated acute GVHD with mAb therapy targeting IL2RAb and soluble TNFAb: single-center experience and systematic review. Am J Transplant 2018; 18:3007-3020.

45. Ambrose $T$, Holdaway $L, S$ mith $A$, et al. The impact of intestinal transplantation

-1. on quality of life. Clin Nutr 2019; doi: 10.1016/j.clnu.2019.08.023. [Epub ahead of print]

This study provides an overview on QoL after intestinal transplantation.

46. Roskott AM, Groen $H$, Rings $E H$, et al. Cost-effectiveness of intestina transplantation for adult patients with intestinal failure: a simulation study. Am J Clin Nutr 2015; 101:79-86.

47. Canovai E, Ceulemans L, Peers G, et al. Intestinal transplantation is cost effective in the treatment of complicated intestinal failure. Transplantation 2017; $101($ S6-2):S90.

48. Lauro A, Matsumoto CS, Marino IR, Berghella V. A review on pregnancy after intestinal transplantation. J Matern Neonatal Med 2017; 30:205-212. 